\title{
EMULATION OF SERIES AND SHUNT REACTOR COMPENSATION
}

\author{
H. Amreiz \\ London College UCK, \\ Division of Engineering \\ London, UK \\ h.amreiz@1cuck.uk
}

\author{
A. Janbey \\ London College UCK, \\ Division of Engineering \\ London, UK \\ a.janbey@1cuck.ac.uk
}

\author{
M. Darwish \\ Brunel University \\ Electronic and Computer Engineering \\ London, UK \\ mohamed.darwish@brunel.ac.uk
}

\begin{abstract}
In the case of large systems of transmission lines with multiple generators which are connected in parallel, sometimes it is essential to use a series reactor in order to prevent large current flow if a short-circuit occurs. This approach protects the conductors of the transmission line and switching circuits from any damage which might result because of the high currents and also the forces, which can be produced in the case of a short circuit. Shunt reactors are connected in parallel with the transmission line or the other loads. A series reactor is usually connected between the load and the source.

The emulator of the transmission lines used in this paper is HVAC transmission line which is $180 \mathrm{~km}$ long. The transmission line can be used in the emulator as a 3-phase of $180 \mathrm{~km}$ length or as a single phase transmission line which is $540 \mathrm{~km}$ long. The transmission line is divided into $6 \pi$ sections and each $\pi$ section is $30 \mathrm{~km}$ long. The line inductance is examined for every $30 \mathrm{~km}$ and the line capacitance is for every $15 \mathrm{~km}$. The line parameters (RLC) of the $400 \mathrm{kV}$ transmission line are: $0.02978 \Omega / \mathrm{km}, 1.06$ $\mathrm{mH} / \mathrm{km}$ and $0.0146 \mu \mathrm{F} / \mathrm{km}$, respectively. The actual power which is carried by the transmission line is $250 \mathrm{w}$.
\end{abstract}

Keywords: transmission line, series reactor, shunt reactor, shunt capacitor, shunt compensation, series compensation.

\section{INTRODUCTION}

In the early days, generation, transmission and distribution of electricity were in most cases DC current. DC systems posed problem which is the fact that the level of the voltage was difficult to change. This is usually achieved by using rotating machines which means the costs will greatly increase. However, nowadays in large power systems AC power systems is used more often than DC systems. At the production power station, electricity is transmitted at very high voltages in order to reduce losses and consequently it has to be stepped down at the substation so that it can be used by the different customers. In the design and operation of transmission lines, it is a fact that the main factors of these lines are the voltage drop, the losses of the lines and the efficiency of transmission lines. It is also a fact that the main parameters that can affect transmission lines are the resistance, the inductance and the capacitance of the transmission line. This paper will present the results of the emulations of series and shunt reactor compensation as well as shunt capacitor compensation of the transmission lines under loading condition.
The emulator used in this paper can be used to investigate 9 simulating experiments. In a previous paper (1) the emulator was used to investigate the Ferranti effect, the $A B C D$ parameters of $400 \mathrm{kV}$ HVAC transmission line and the surge impedance loading. This paper will investigate the shunt reactor compensation, shunt capacitor compensation, and series reactor compensation,

\section{FACTORS AFFECTING THE TRANSMISSION LINE}

It is a fact that the performance of the AC power systems depends largely on the performance of transmission line in the system. On the other hand the performance of the transmission lines depends on the series resistance $\mathrm{R}$, inductance $\mathrm{L}$, the shunt capacitance $\mathrm{C}$ and the conductance $\mathrm{G}$. Resistance $\mathrm{R}$ is the opposition to the flow of the current and inductance $\mathrm{L}$ arise because the conductor is carrying current which is surrounded by the force of the magnetic lines around the conductor. The capacitance of the line $\mathrm{C}$ is due to the fact that the conductor that carries the current will form a capacitor with the earth. This capacitance will always be at lower potential and as a result the conductor and the earth will form the parallel plates of the capacitor. The air between these parallel plates will form the dielectric material. The shunt conductance $\mathrm{G}$ is because of the leakage current which is present over the surface of the insulators especially in bad weather conditions. Furthermore the line impedance will exert the voltage drop in the line in quadrature with the current in the conductor. This is usually found by utilizing the equation $2 \pi \mathrm{fLI}$ volts. In this equation $\mathrm{f}$ is the frequency of the supply in $\mathrm{Hz}$, the inductance $\mathrm{L}$ in each conductor is in Henry and I is the current in the conductor in Ampere. The line capacitance in transmission lines result in a current which is called the charging current. This current is in quadrature with the voltage. The charging current reaches its maximum value at the sending end of the line and it will keep decreasing and eventually will become zero at the receiving end of the transmission line. The value of the charging current IC can be calculated at the sending end using the equation $2 \pi f C V s$. The term $\mathrm{f}$ is the frequency of the supply in $\mathrm{Hz}, \mathrm{C}$ is the capacitance in Farad and Vs is the voltage at the sending end. The shunt conductance is in parallel with the system and consequently the leakage currents in the transmission lines are small. It is for this reason that the shunt conductance $G$ is 
ignored in the calculations. The reactive drop $(2 \pi \mathrm{fLI})$ and the charging current $(2 \pi \mathrm{fCVs})$ are proportional to the frequency of the supply (5). The reason for this is because of the fact that they exert strong influence on the performance when the frequency is high. In addition to this it is well known that the effects of reactance of the overhead line are very important. This is so because of the wide space between the conductors. On the other hand, the effects of the reactance in the underground line are small and those of the capacitance have the largest contributions (6). The actual generating and the receiving stations are shown in figure 1 and 2 respectively (7). Schematics of the units of the actual connections of the apparatus are shown in the connection diagrams in figure 3, figure 8 and figure 10 .

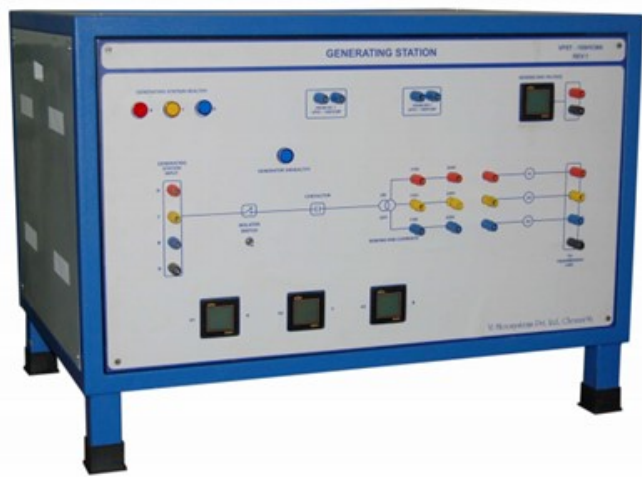

Figure 1. Generating Station of emulator

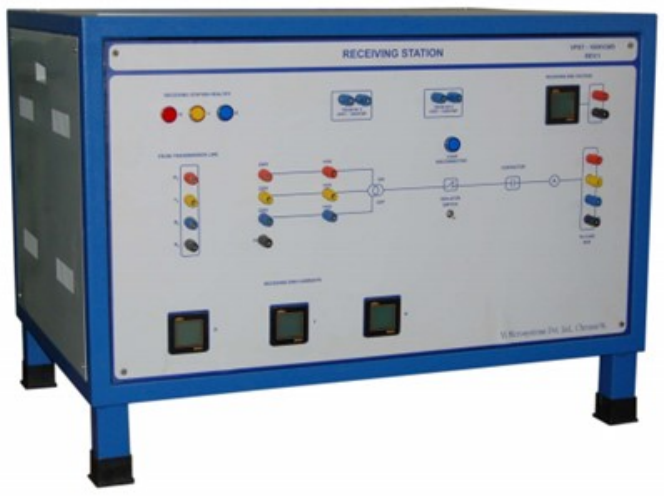

Figure 2. Receiving station of simulator

\section{1-SHUNT REACTOR COMPENSATION}

The aim of this experiment is to analyse and control the increased voltage at receiving end due to light load or no load voltage. Shunt reactors are installed at the sending end and receiving end of long transmission lines. Sometimes they are also employed at the intermediate switching sub stations to absorb the leading Vars supplied by shunt admittances during small loads or no loads (8). During low loads the receiving end voltage tends to increase due to the effect of shunt admittance of line to control the voltage. Line to ground capacitance should be compensated and this is achieved by switching the shunt reactors. During high loads the reactance current drop increases and the voltage tends to fall below its rated value and consequently the shunt reactors are switched off. The connection of the experiment is shown in figure 3 with actual schematics of the apparatus shown in figures 1, 2, 5, 6 and 7 in this paper.

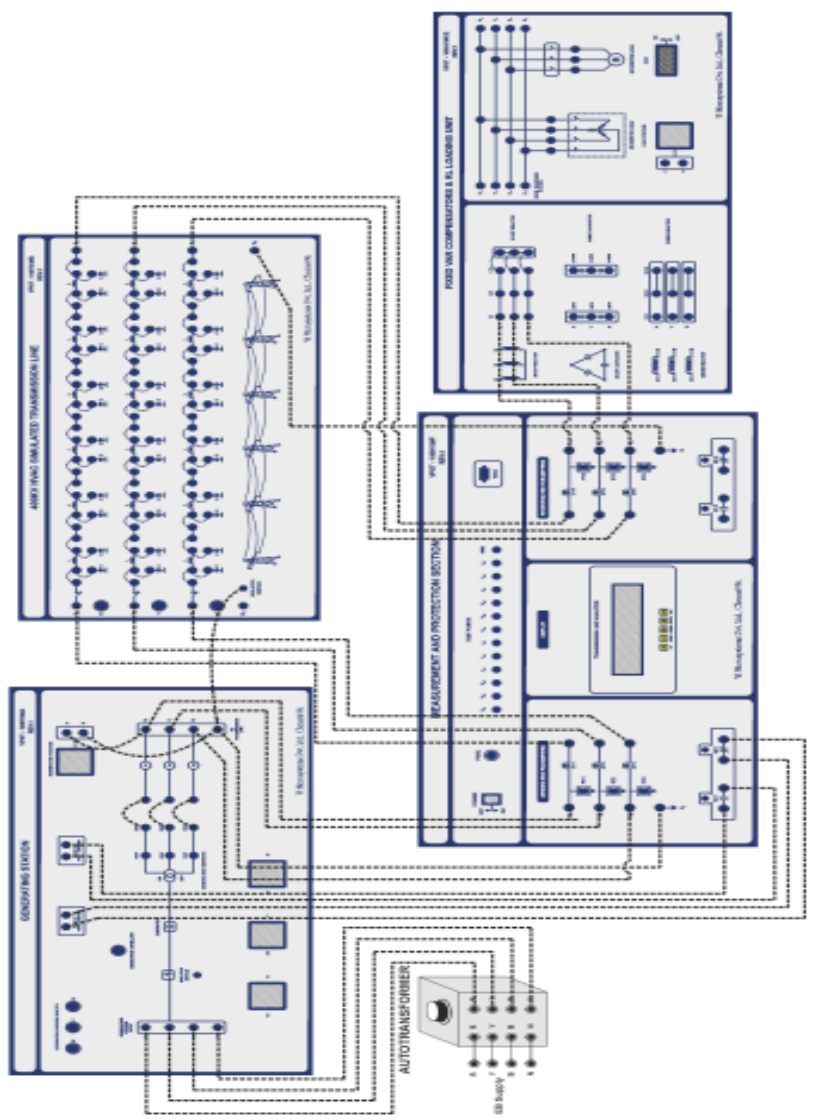

Figure 3 Connections of shunt reactor compensator (Schematics of actual apparatus shown in figures 1,2,5,6, and 7 shown in this paper)

A shunt reactor is an electrical inductor which is used in the cases of high voltage transmission lines in order to stabilize the voltage during changes of the load. In conventional cases shunt reactor has fixed ratings and it can be connected continuously to the transmission line or it can be switched in and out and this depends on the type of load.

In the case of a three phase shunt reactor it is connected to $400 \mathrm{kV}$ or above for capacitive reactive power compensation of the transmission line and also to control the over voltage in the system because of load rejection.

The shunt reactors are able to tolerate maximum continuous operating voltage (this is usually $5 \%$ higher than rated voltage when using $400 \mathrm{kV}$ system). This is in the case of normal common power frequency changes and the temperature should not go above $150^{\circ} \mathrm{C}$.

Two commonly used shunt reactors are emulated and these are the gapped core type or the magnetically shielded air core type. These designs keep the impedance of the reactor fixed. The impedance in the transmission line is kept at a constant value 
in order to avoid harmonic current which can be generated because of any over voltage. The result of the simulation of shunt reactor compensation is shown in figure 4 .

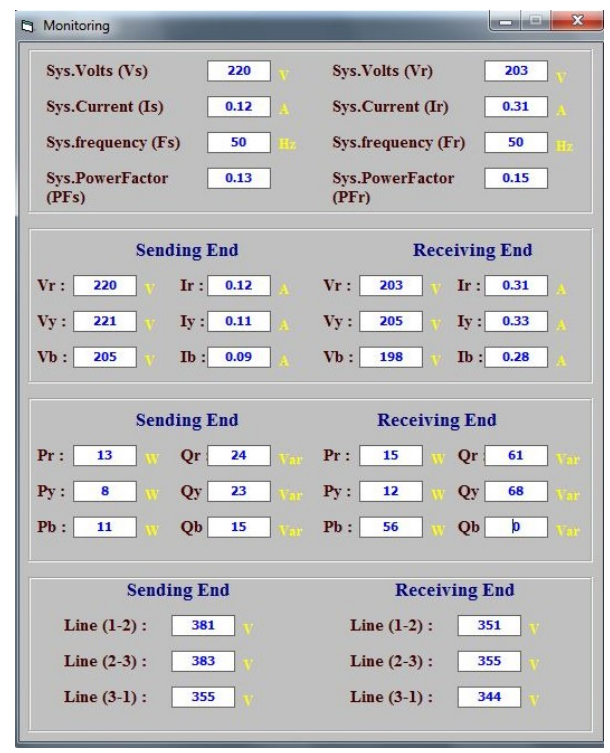

Figure 4. Results of simulation of shunt reactor compensation

The core losses of the shunt reactor are always present in normal operating condition. It is for these reasons that the design is carefully optimised in order to reduce core losses.

The losses of the shunt reactor are measured at the rated voltage and frequency. However in the case of the use of very high voltage shunt reactor, it can be quite difficult to carry out testing during measurement of losses because of the high voltage value. This problem is avoided, by measuring the losses at a voltage which is lower than the voltage of the reactor. The actual transmission line unit used for these experiment is shown in figure 5. The Measurement and protection unit is shown in figure 6 and the compensator unit and RLC loading sections are shown in figure 7.

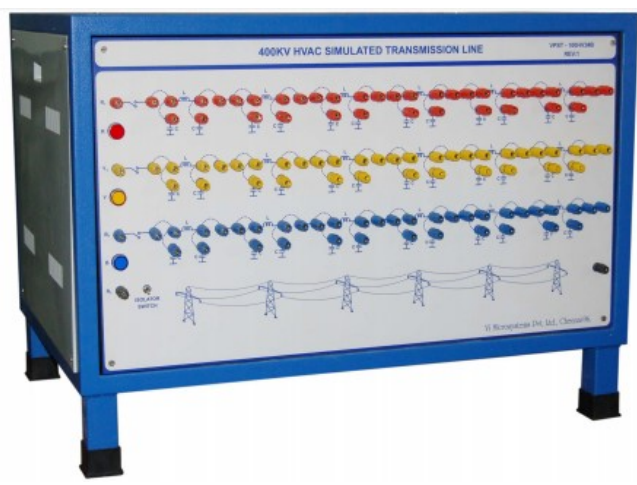

Figure 5. Transmission line unit

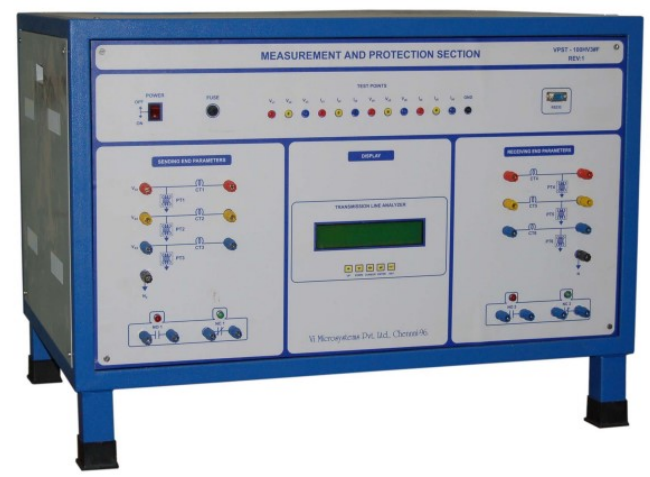

Figure 6.Measurement and protection unit

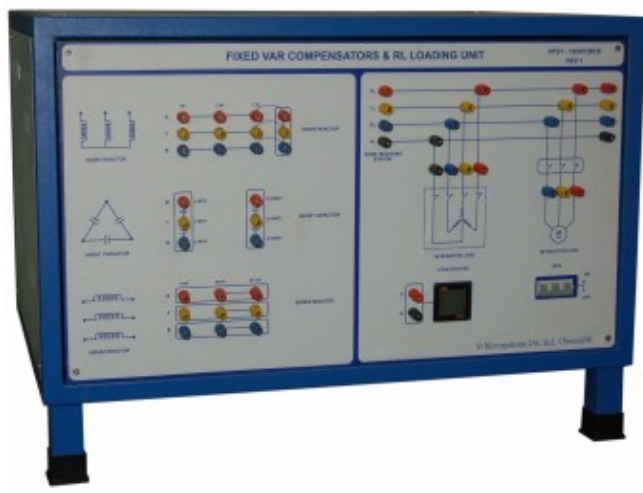

Figure 7. Compensator unit and RLC loading section

\section{2-SHUNT CAPACITOR COMPENSATION}

The aim of this experiment is to control the receiving end voltage during heavy loaded conditions. Shunt Capacitors are connected at the receiving end in order to provide leading Var. Shunt Capacitors are switched on when $\mathrm{kVA}$ demands reactive power increase and voltage of the receiving end is reduced. The switching of the shunt capacitor compensator increases the voltage at the receiving end. Thus it improves the power factor and voltage region which saves energy due to reduction of line losses. It also reduces $\mathrm{kVA}$ demand which in-turn reduces line current. The schematic connections of shunt capacitor compensation are shown in figure 8. These schematics represent the actual apparatus shown in figure 1, figure 2, figure 5 , figure 6 and figure 7 in this paper. 


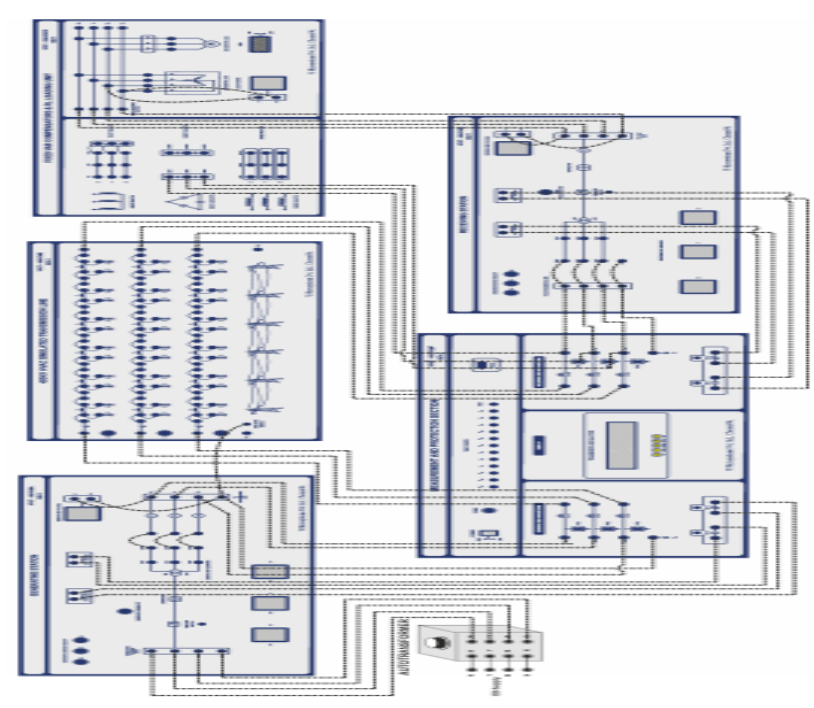

Figure 8. Connections of shunt capacitor compensation (Schematics of actual figures of the apparatus shown in figures $1,2,5,6$, and 7 in this paper)

Shunt reactors are used in compensation very effectively against the capacitive behaviour in high voltage transmission lines. After load rejection or light load conditions, a resonance occurs because of the capacitive characteristics of these transmission lines. This leads to failure of the reactor which can damage the interior insulation of high voltage apparatus which is connected to the transmission line. Premature damage to the insulations are serious faults in resonance voltages. In order to investigate these faults, different cases are examined which includes total disconnection of the transmission line, single and double pole operation of breakers, and short circuit faults on the de-energized line. These simulations utilize two different knee points of the saturation of the reactor. Different solutions including neutral reactors and resistors, transposition of the circuits and capacitor bank are examined in the worst case scenario in order to control the resonance overvoltage. It is shown here that when the shunt reactor is not accurately determined, no single solution can deal effectively with the resonance phenomenon.

In the cases of shunt compensation, parallel connection is used with the transmission lines of the power system which works as a controllable current source. In this case a reactive current is fed into the transmission line in order to keep the voltage constant by changing the shunt impedance. It is because of this that the active power which can be transmitted is increased but this results in increasing the demand of the reactive power.

\section{$i$-Shunt capacitive compensation.}

The shunt capacitive compensation is used in order to improve the power factor. When there is an inductive load which is connected to the transmission line, the power factor lags because of the lagging current of the load. In order to compensate for this, a shunt capacitor must be connected and which draws current leading to the source voltage and consequently leads to improving the power factor.
ii-Shunt inductive compensation.

The shunt capacitive compensation is used in two cases: when charging the transmission line or for very low load at the receiving end of the transmission line. Because of the low load or the nonexistence of any load, the current in the transmission line is very low. The Shunt capacitance in the transmission line results in voltage amplification (This is called the Ferranti effect). The receiving end voltage $\left(V_{\mathrm{r}}\right)$ can become twice as much as at the sending end voltage $\left(V_{\mathrm{s}}\right)$ (This is usually common in long transmission lines which is the case in our emulator). In order to compensate for this, shunt inductors are connected with the transmission line. The Simulation results of shunt capacitor compensation are shown in figure 9.

The compensation maintains a voltage, $V_{\mathrm{c}}$, equal to the bus bar voltage such that $V_{\mathrm{s}}=V_{\mathrm{r}}=V_{\mathrm{c}}=V$. Each half of the line is represented by a $\pi$ equivalent circuit. In order to absorb the reactive power for the two extreme sections synchronous machines can be connected at the two ends. This will result in the compensator supplying or absorbing only the reactive power for the middle section of the transmission line. It is clear here that the compensator does not consume real power because the compensator voltage and current are in quadrature. The compensator varies its admittance continuously in order to keep the midpoint voltage $V_{\mathrm{c}}=V$. In the steady state, the line is divided into two independent halves. In addition to this, the power which is transferred from the sending end to the midpoint is actually equal to the power transferred from the midpoint to the receiving end.

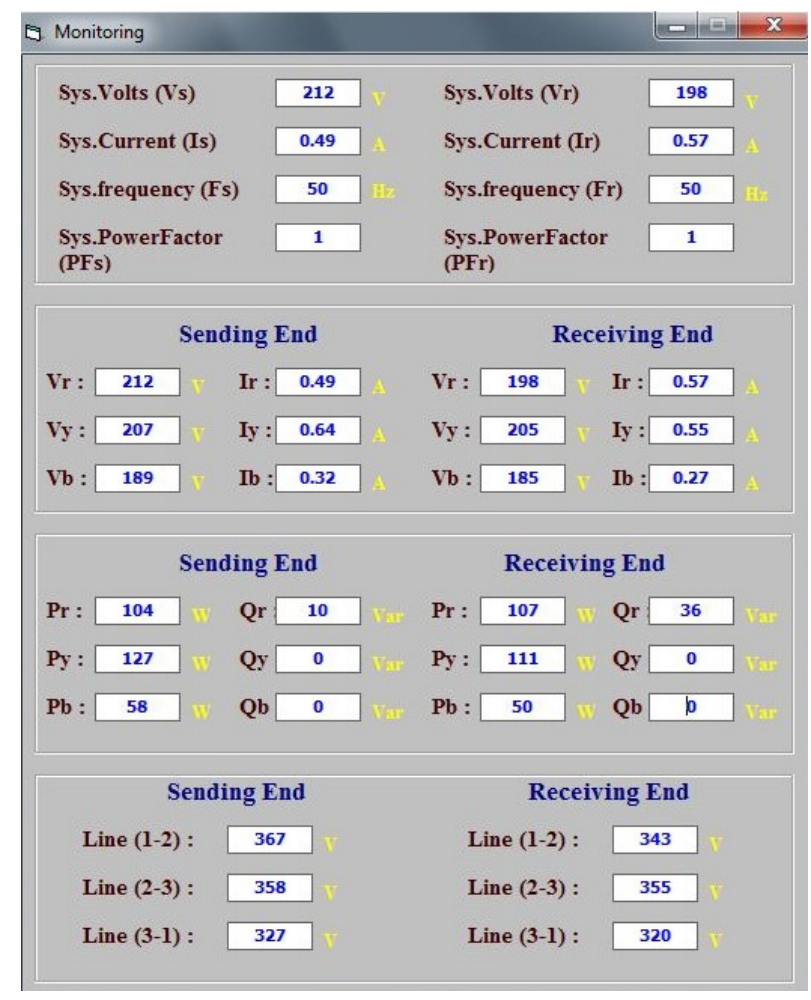

Figure 9. Simulation results of shunt capacitor compensation 


\section{3-SERIES REACTOR COMPENSATION}

The aim of the experiment is to control the fault current at the receiving end during fault condition. The series reactors are connected in series with the power system to reduce the fault current.These reactors can be connected at anywhere in the power system. There are reactors, which can be connected at the generator ends called generator reactors for the protection from fault current. Similarly there are reactors for the transformer protection and also transmission line protection. The reactor is nothing but a huge amount of inductor which can withstand huge fault currents. The connection of the series reactor compensation are shown in figure 10 . These schematics represent the actual units of the apparatus shown in figure $1,2,5,6$, and 7 in this paper. Series compensation is used in order to improve the system voltage and this is achieved by connecting a capacitor in series with the transmission line. In series compensation, reactive power is connected in series with the transmission line in order to improve the impedance of the system. It also improves the level of power transfer of the line and it is mostly used in extremely high voltage lines.

Series compensation has several advantages such as increasing the capacity of transmission, improving the stability of system, controlling the voltage regulation and ensuring better division of the loads between the parallel feeders.

It also improves the voltage profile as well as providing voltage support for long HVAC transmission lines. This is acheived by introducing capacitance in the the transmission line. Series compensation also increases the flow of the power and improves the stability of power system by reducing the impedance of the line. The improvement of the stability of the power system results in additional capabilty of the power transfer which occurs during any needed transient event. Series compensation also minimizes the requirements of land which is needed for the system installation. Furthermore series compensation decreases environmental impact by stopping any needs for any new infrastructure. The results of the emulations of the series reactor compensations are shown in figure 11 .

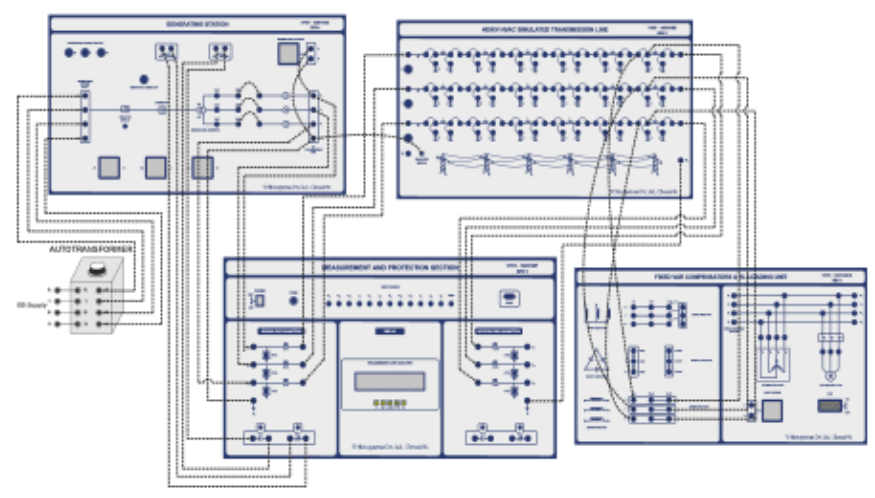

Figure 10. Series reactor compensation ( Schematics of actual units of the apparatus shown in figure 1, $2,5,6$,and 7 in this paper)

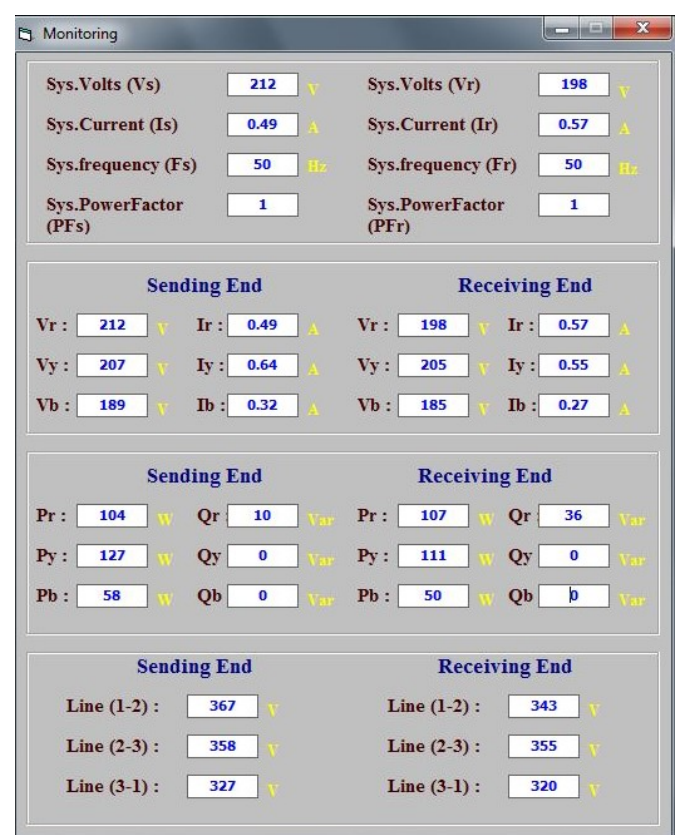

Figure 11. Simulation results of series reactor compensation

For the same power transfer and for equal values of voltage at the sending and receiving end in the case of the series impedance line, the phase angle $\delta$ is less in this case than that of the case when the system is for uncompensated line. This smaller value of $\delta$ results in better stability. Series capacitors are used in the transmission lines in order to improve dividing the load between the parallel lines. This means new line with a transfer of power which is a large portion of power in parallel with the existing line and becomes very difficult to put load on the new line without overloading the existing line. In this situation the series compensation will reduce the series reactance and consequently dividing the load between the parallel circuit will become easier. The division of the load will increase the power transfer of the the transmission line and consequently will reduce power losses. In the case of series capacitor, there is a consequent variation in the reactive power as the load current changes and as a result a fall in the level of the voltage will occur because of the sudden change of the load which is corrected immediately.

The position of the series capacitor is dependent on the technical and economic factors of the line. The series capacitor can be positioned at the sending end or receiving end or even at the center of the line and in some cases it can be positioned at more than two points. The position of the capacitor is decided by the level of compensation as well as the characteristic of the line. Positioning the capacitors at the terminal facilitates maintenance, but on the other hand the overvoltage that appears at the terminals of the capacitors when a fault occurs results in overstressing the capacitor.The capacitors are positioned in the intermediate switching station but only in the cases of long transmission lines. Furthermore, The positioning of the capacitor in the middle of the line decreases the ratings of the capacitor. The banks of the 
capacitor are made up of small units which are connected in series or parallel, or even series and parallel in order to obtain the required ratings of the volatage as well as Var rating. In the case when a fault or overload occurs, a large current flows in the series capacitor of the transmissiion line. Consequently this will result in large voltage drop in the transmission line. In order to protect the capacitors in such cases, surge diverter are connected across the terminals of the capacitor. In addition to this measure a circuit breaker is also connected in parallel with the capacitor in the transmission lines.

\section{PROBLEMS ARISING IN SERIES CAPACITOR}

It must be stated here that there are some problems that arise in series capcitor which include the following:

In the case of frequencies lower than the power frequency, the series compensated line results in series resonance which is known as sub-synchronous resonance. Sub-synchronous resonance generally occurs when faults arise or during the switching operation. However this type of resonance is alleviated by using a filter, passing the series capacitor bank under this type of resonace or tripping the generator.

It is a fact that series capacitors produce high recovery voltages at the contacts of the breakers. If the level of the compensation and positioning of the capacitors are not accurately chosen, the distance relays will not work properly. If switching of a transformer which is unloaded at the end of a series compensation of the transmission, line non-linear resonance or ferro resonance might occur and consequently uninterrupted oscillations arise.

The frequency of these uniterrupted oscillations is reduced by the use of shunt reactors across the capacitors or even short circuiting the capacitors temporarily. It could be said that series capacitors produce more net increase of voltage which produces more voltage drops in the system.

\section{Conclusions}

An emulator is used to test an inductive shunt reactor in the cases of high voltage transmission lines in order to stabilize the voltage during changes of the load. In the case of a three phase shunt reactor it is connected to $400 \mathrm{KV}$ or above for capacitive reactive power compensation of the transmission line and also to control the over voltage in the system because of load rejection. The shunt reactor is able to tolerate maximum continuous operating voltage (this is usually $5 \%$ higher than rated voltagewhen using $400 \mathrm{KV}$ system). This is in the case of normal common power frequency variations and without going above a temperature of $150^{\circ} \mathrm{C}$.

As predicted, the switching of the shunt capacitor compensator increased the voltage at the receiving end and resulted in improvement of the power factor and voltage region which saves energy due to reduction of line losses. It also reduced $\mathrm{kVA}$ demand which in turn reduced the line current. Load rejection or light load conditions resulted in resonance because of the capacitive characteristics of these transmission lines. In practise this of course leads to failure of the reactor which can damage the interior insulation of high voltage apparatus. Premature damage to the insulations were investigated and in order to do, different cases are examined which included total disconnection of the transmission line, single and double pole operation of breakers, and short circuit faults on the deenergized line.

In pratical series compensation, reactive power was connected in series with the transmission line in order to improve the impedance of the system. This has resulted in improving the level of power transfer of the line and has also improved the voltage profile as well as providing voltage support for long HVAC transmission lines. This was acheived by introducing capacitance in the the transmission line. This series compensation also increased the flow of the power and improved the stability of power system by reducing the impedance of the line.

\section{References}

[1] H. Amreiz, Mohamed Darwish, Al Janbey, "Simulation of hvac transmission line, ".UPEC2019. Bucharest, Romania 2019.

[2] Electric Power Engineering Education Resources 2005-2006 IEEE Power Engineering Society Committee Report. The Power Engineering Education Committee (PEEC) Task Force on Educational Resources.

[3] J. McCalley ; L. Bohmann ; K. Miu ; N. Schulz. IEEE Transactions on Power Systems Year: 2008 Volume: 23 , Issue: 1 .Pages: $1-24$

[4] Guangye Si ; Julien Cordier ; Ralph M. Kennel. Extending the Power Capability With Dynamic Performance of a Power-Hardware-in-theLoop Application-Power Grid Emulator Using "Inverter Cumulation" IEEE Transactions on Industry Applications Year: 2016 Volume: 52, Issue: 4Pages: $3193-3202$

[5] D. Goldsworthy, T. Roseburg, D. Tziouvaras, and J. Pope, "Controlled Switching of HVAC Circuit Breakers: Application Examples and Benefits," proceedings of the 34th Annual Western Protective Relay Conference, Spokane, WA, October 2007.

[6] C. Portela and M. tavaros. "Modelling, simulation and optimization of transmission lines. Applicability and limitation of some used procedures.

[7] Manul of hvac transmission line analyzer. VPST 100HV3. Vi Microsystems Pvt. Ltd.

[8] Z. Gajić, B. Hillström, and F. Mekić, "HV Shunt Reactor Secrets for Protection Engineers," proceedings of the 30th Annual Western Protective Relay Conference, Spokane, WA, October 2003.

[9] CIGRE WG 13.07, "Controlled Switching of HVAC Circuit Breakers: Guide for Application," Part 2, ELECTRA, No. 185, August 1999.

[10] W. D. Stevenson, Jr., Elements of Power System Analysis, 4th ed. McGraw-Hill, 1982. 
